\title{
Reliability of the Path of the Sciatic Nerve, Congruence between Patients' History and Medical Imaging Evidence of Disc Herniation and Its Role in Surgical Decision Making
}

\author{
Keyvan Mostofi ${ }^{1}$, Reza Karimi Khouzani ${ }^{2}$ \\ ${ }^{1}$ Department of Neurosurgery, Centre de Chirurgie Endoscopique de Rachis, Clinique Bel Air, Bordeaux, France \\ ${ }^{2}$ Department of Neurosurgery, International Neuroscience Institute, Hannover, Germany
}

Study Design: The prevalence of disc herniation is estimated to be about 100000 new cases per year in France and disc herniation accounts for $25 \%$ to $30 \%$ of surgical activity in Departments of Neurosurgery. Classically, sciatica is expected to follow its specific dermatome $-\mathrm{L} 5$ or $\mathrm{S} 1-$. In clinical practice, we regularly encounter patients showing discrepancy between clinical sciatica and imaging findings.

Purpose: The aim of this paper is to review the medical concept and management of sciatica pain in patients showing this discrepancy. Overview of Literature: To the best of our knowledge, this subject has not yet been discussed in the medical literature.

Methods: The medical records of 241 patients who were operated on for L5 or S1 sciatica caused by disc herniation were reviewed. Results: We found an apparent clinicoradiological discrepancy between sciatica described by patients on one side and magnetic resonance imaging (MRI) finding on the other side in $27(11.20 \%)$ patients. We did not find any other abnormalities in the preoperative and postoperative period. All of these patients underwent lumbar discectomy via posterior interlaminar approach. Three months after surgery, 25 patients (92.59\%) had been totally relieved of sciatica pain. Two patients $(7.41 \%)$ continued to experience sciatica in spite of the surgery.

Conclusions: The discrepancy between clinical sciatica and disc herniation level on MRI is not rare. Management of this discrepancy requires further investigation in order to avoid missing the diagnosis and treatment failure.

Keywords: Sciatica; Radiculopathy; Low back pain; Intervertebral disk degeneration

\section{Introduction}

Classically, sciatica from L5 and S1 nerve roots caused by a non foraminal posterolateral L4-L5 and L5-S1 herniated disc, respectively is manifested as radiculopathy along the path of the affected nerves. Sciatica from the L5 nerve root spreads over the buttock, posterolateral part of the thigh, posterolateral part of the leg, passes over the instep and ends at the big toe. Sciatica from the S1 nerve root covers the buttock, posterior part of the thigh, posterior part of the leg, popliteal fossa area, heel, lateral edge of the foot, and radiates all the way to the little toe. In this article, this classical medical concept is discussed based on the practical experience of the authors in the surgical

Received Aug 3, 2014; Revised Sep 9, 2014; Accepted Sep 18, 2014

Corresponding author: Keyvan Mostofi

Department of Neurosurgery, Centre de Chirurgie Endoscopique de Rachis, Clinique Bel Air,

138, Avenue de la République, 33000, Bordeaux, France

Tel: +33-5-57-10-72-95, Fax: +33-5-57-10-72-93, E-mail: keyvanmostofi@yahoo.fr 
treatment of lumbar disc herniations. An almost ten years of experience illustrates the fact that despite these conventional conceptions, we regularly face a different reality: the reality of discrepancy between the clinical sciatica and imaging findings. This discrepancy is even more problematic when a surgical decision has to be made. In this paper, we discuss our experience with the therapeutic approach for management of sciatica in patients with this clinicoradiological discrepancy. In clinical practice, we frequently need to manage patients with sciatica from L5 with postérolatérale L5-S1 disc herniation on magnetic resonance imaging (MRI) imaging or sciatica from S1 with L4-L5 posterolateral disc herniation on MRI findings. The cases of foraminal herniated disc, lumbosacral transitional vertebrae-sacralisation of L5 and lumbarisation of S1 - and conjoined nerve root were not included in this study.

\section{Materials and Methods}

We reviewed the medical records of 241 patients who were operated on for L4-L5 and L5-S1 disc herniation by authors working in various French and French West Indies hospitals in the last ten years. All of these patients underwent lumbar discectomy via posterior interlaminar approach for non foraminal lumbar disc herniation. In
27 (11.20\%) of the 241 patients, an apparent clinicoradiological discrepancy was found between sciatica described by patients on one side and MRI finding on the other side (Figs. 1, 2). All of the patients underwent magnetic resonance imaging scan. Among them, 11 patients had undergone an initial computed tomography (CT) scan. Eighteen patients had undergone electromyography (EMG) before surgical decision making. In all of the patients, conservative management had failed and despite this discrepancy, a decision to operate was taken after obtaining consent of the patients on explaining them that this clinicoradiological discrepancy may yield uncertain and random surgical results.

\section{Results}

Twenty-two patients $(81.48 \%)$ reported that their sciatica pain had been totally relieved at six weeks after surgery, and 25 patients $(92.59 \%)$ reported that their sciatica pain had been totally relieved at three months after surgery. Five patients experienced slight relief from sciatica pain at six weeks after surgery, and among them, 3 patients experienced total relief from sciatica pain at 3 months after surgery. Therefore, after 3 months of surgery, 25 (92.59\%) patients reported that their sciatica pain had been re-

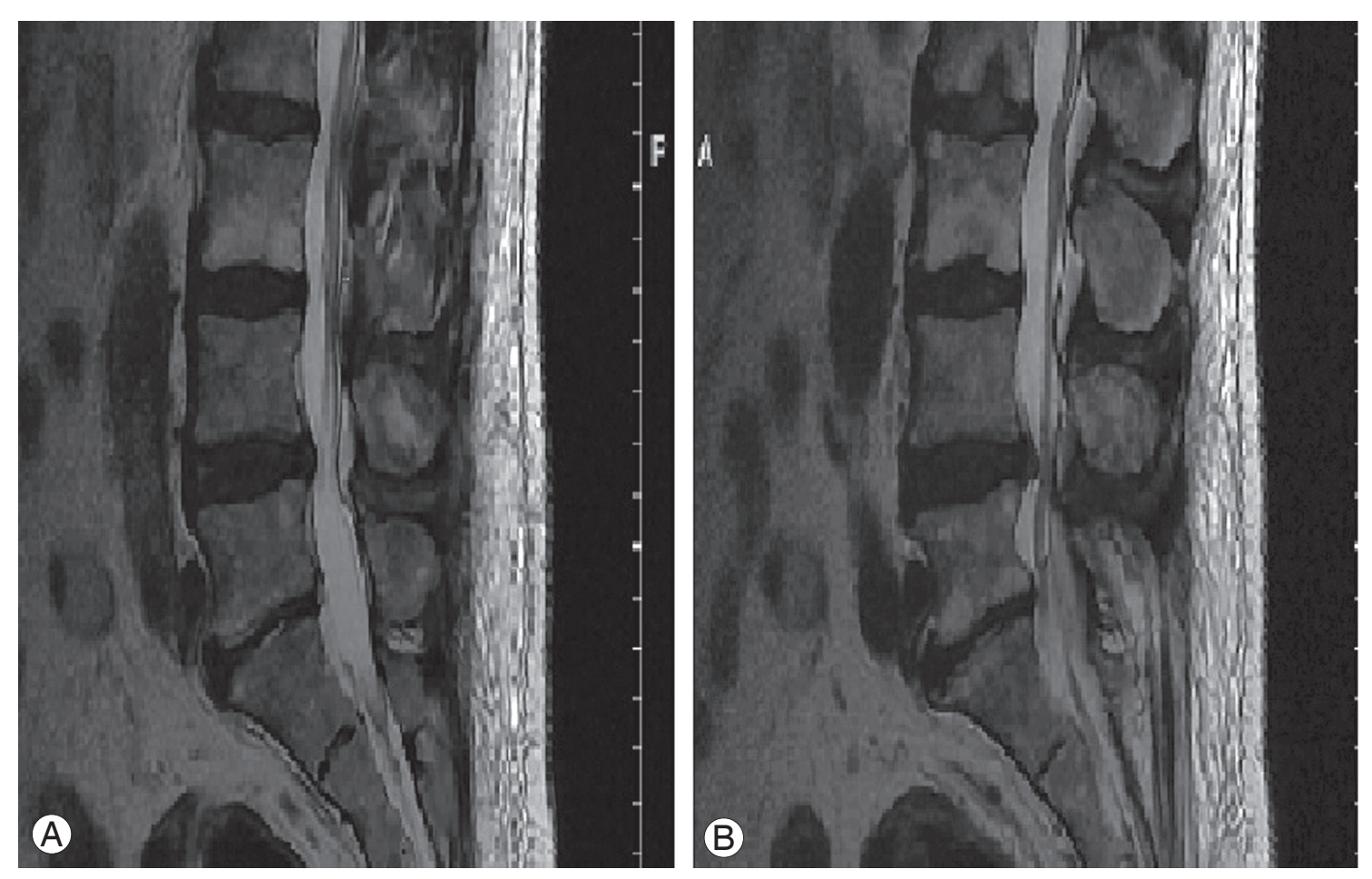

Fig. 1. (A, B) Axial magnetic resonance imaging scan showing L5-S1 disc herniation in a patient and L5 sciatica. 


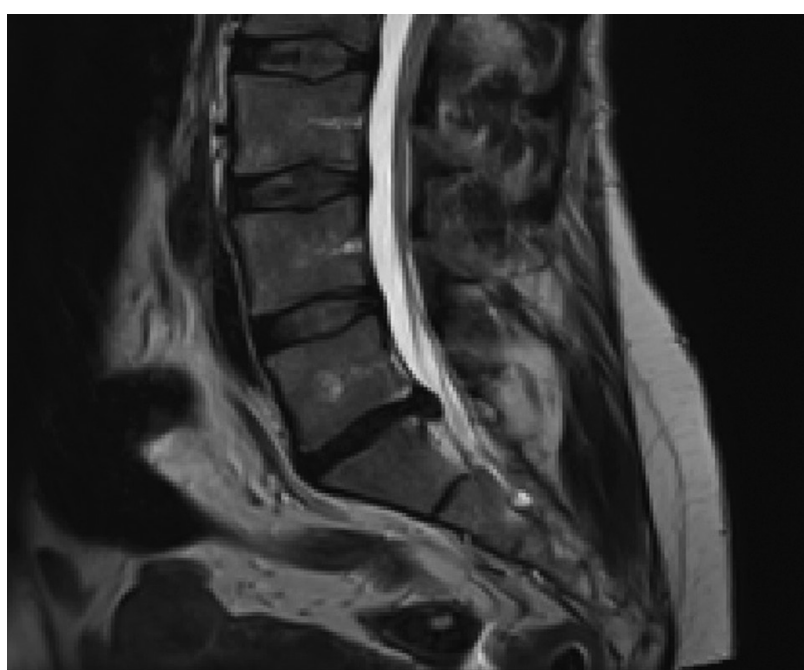

Fig. 2. Axial magnetic resonance imaging scan showing $L 4-5$ disc herniation in a patient with $\mathrm{S} 1$ sciatica.

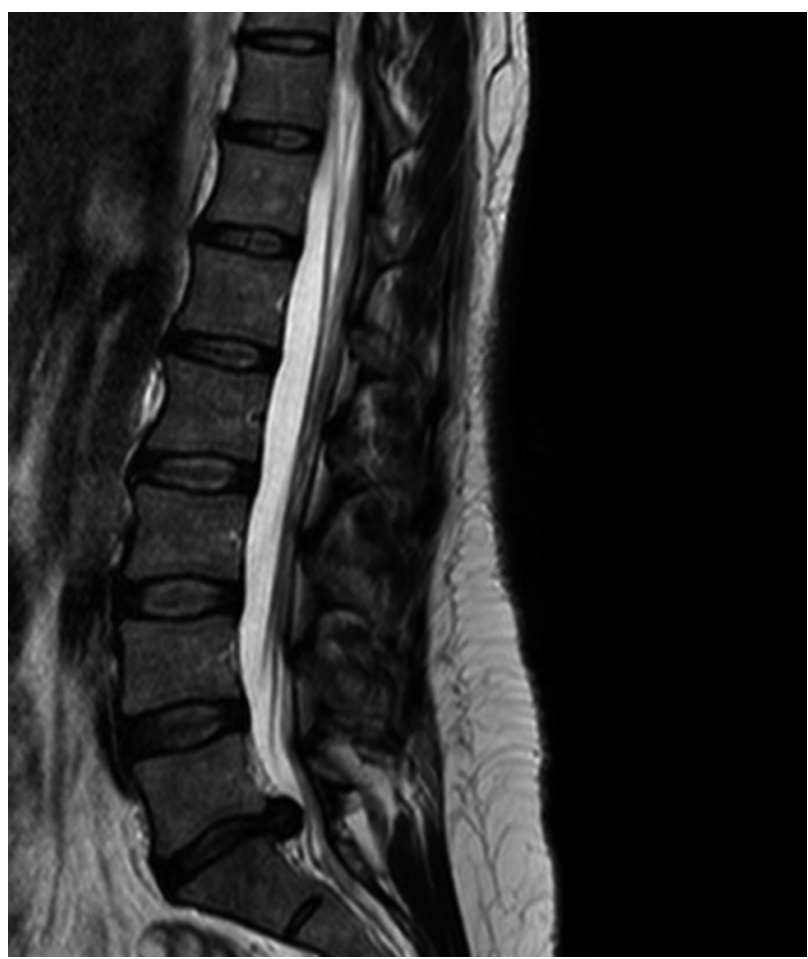

Fig. 3. Preoperative axial magnetic resonance imaging scan of a patient who had persistent symptoms after 3 months of the operation.

lieved. Two patients (7.41\%) continued to experience pain at 3 months after surgery (Fig. 3). Lasègue's sign became negative in 26 (96.29\%) patients at 3 months after surgery. Table 1 presents patient characteristics in the preoperative and postoperative period. The patients who had not undergone CT scan benefited from lumbosacral spine Xray that helped to ensure that there were no lumbosacral
Table 1. Patient's characteristics and data in preoperative and postoperative period

\begin{tabular}{|c|c|}
\hline Characteristic & Value \\
\hline Average age & $43.81(22-78)$ \\
\hline \multicolumn{2}{|l|}{ Herniation level } \\
\hline L4-L5 & 15 P. (55.5\%) \\
\hline L5-S1 & 12 Р. $(44.4 \%)$ \\
\hline \multicolumn{2}{|l|}{ Medical imaging } \\
\hline MRI scan & $27 \mathrm{P}$. \\
\hline TDM & $11 \mathrm{P}$. \\
\hline \multicolumn{2}{|l|}{ Lasègue's sign } \\
\hline Preoperative period & $27 \mathrm{P}$. \\
\hline 6 wk after surgery & $3 \mathrm{P}$. \\
\hline 3 mo after surgery & $1 \mathrm{P}$. \\
\hline Electromyography & $18 \mathrm{P}$. \\
\hline \multicolumn{2}{|l|}{ Sciatalgie } \\
\hline \multicolumn{2}{|l|}{ Preoperative period } \\
\hline Intense & $27 \mathrm{P}$. \\
\hline Moderate & $O P$. \\
\hline Relieved & $O P$ \\
\hline \multicolumn{2}{|l|}{6 wk } \\
\hline Intense & OP. \\
\hline Moderate & $5 \mathrm{P}$. \\
\hline Relieved & $22 \mathrm{P}$. \\
\hline \multicolumn{2}{|l|}{$3 \mathrm{mo}$} \\
\hline Intense & $O P$. \\
\hline Moderate & $2 P$ \\
\hline Relieved & $25 \mathrm{P}$. \\
\hline
\end{tabular}

MRI, magnetic resonance imaging; TDM, tomodensitometry; P., patient.

transitional vertebrae-sacralisation of L5 and lumbarisation of S1-. During surgery, the nerve root was carefully checked from its emergence to spinal ganglion to ensure that there was no conjoined nerve root. None of the patients had lumbosacral transitional vertebrae or conjoined nerve root. We did not find significant bulging or herniated disc at another level of the lumbar spine in the patients.

\section{Discussion}

The sciatic nerve is the longest and widest single nerve in the human body. It practically innervates all muscles of the back of the thigh, and those of the leg and foot. It is derived from the L4 to S3 segments of the sacral plexus. 
It divides into two terminal branches: the tibial nerve and the common fibular nerve. Degenerative diseases of the lumbar spine, especially lumbar herniation, directly affect the nerve roots which form the sciatic nerve. There is an increasing prevalence of degenerative diseases of the lumbar spine, mainly lumbar disc herniation, particularly in industrialized countries [1]. They are one of the major causes of cessation of work and healthcare expenditure [2-4].

In a survey undertaken in 2004 by the National Institute of Statistics and Economic Studies in France, the prevalence of degenerative diseases of the lumbar spine was estimated to be $7.9 \%$ in men and $7.5 \%$ in women. They are a cause of social handicap and numerous work stoppages [5]. The prevalence of disc herniation is estimated to be about 100,000 new cases per year in France. Disc herniation leads to 37,000 surgeries per year and accounts for $25 \%$ to $30 \%$ of surgical activity in Departments of Neurosurgery [6]. The diagnosis of sciatica is relatively easy and can be practically made immediately after taking the patient's medical history. Clinical examination and imaging confirm the already suspected diagnosis. In our current practice of neurosurgery, we regularly encounter abnormalities which can lead to some discrepancies between clinical sciatica and the level of disc herniation. The most frequent causes of the latter are lumbosacral transitional vertebrae and conjoined nerve root [7-10]. Identification of these abnormalities is crucial because mistaken diagnosis may lead to surgical errors and haphazard therapeutic results. Lumbosacral transitional vertebrae are common in the general population with a prevalence of $4 \%-30 \%$ [11]. In their study, Lotan et al. [12] estimated conjoined nerve root's incidence at $5.8 \%$ of microdiscectomies performed. Lumbosacral transitional vertebrae require preoperative investigations to avoid missing the diagnosis. For conjoined nerve root, the diagnosis is made peroperatively. However in some cases, no abnormalities are found but there is a clinicoradiological discrepancy between the sciatica pathway and the level of disc herniation. Concerned practitioners who manage sciatica such as rheumatologists and spine surgeons must deal with this discrepancy to avoid misdiagnosis and therefore an effective treatment. In our experience, we did not observe any discrepancy between L5 or S1 clinical sciatica and L3-L4 disc herniation. Among the 241 patients operated on for L4L5 or L5-S1 disc herniation by the corresponding author, in $27(11.20 \%)$ patients, we found an apparent clinicoradiological discrepancy between sciatica described by patients on one side and MRI finding on the other side without any other abnormality. The cases of foraminal herniated disc were excluded. In the event of any doubt regarding presence of differential diagnosis of sciatica other than disc herniation and when there was no indication for emergency surgery, patients were referred to neurologists and rheumatologists to rule out any other differential diagnosis such as peripheral neuritis, diabetic polyneuropathy, sensory-motor mixed neuropathy, or other rheumatological and neurological diseases.

In this paper, we aimed to estimate the rate of this discrepancy in a population suffering from sciatica. Conceptually, EMG may be a useful examination for identifying the affected and compressed nerve. In our series, 18 patients (66.6\%) had preoperative EMG. Among them, 10 patients had L5 clinical sciatica with a L5-S1 disc herniation on the MRI scan. In 3 patients (30\%), EMG demonstrated signs of $\mathrm{S} 1$ nerve root affection consistent with S1 sciatica. EMG demonstrated 2 cases (20\%) of L5 nerve root affection compatible with MRI scan, and in 5 cases (50\%), it was not significant and contributory. Eight patients had S1 clinical sciatica with a L4-L5 disc herniation. Among them, EMG demonstrated signs of L5 nerve root affection in 4 patients (50\%), and signs of S1 nerve root affection in 1 patient (12.5\%), and it was not contributory in 3 patients (37.5\%) (Table 2).

All of the EMGs were performed at least 6 weeks after the onset of sciatica. EMGs were carried out in different centres and by different physicians. During our initial experience, we used to seek assistance from EMG frequently. Over the years, these contradictory results prompted us to not request EMG systematically, to avoid it progressively, and to give preference to clinical signs other than in exceptional cases. Mondelli et al. [13] found abnormal results on EMG in at least one muscle supplied by femoral and sciatic nerves in $42 \%$ of patients with sciatica.

\section{Conclusions}

Our study shows that $11 \%$ of patients suffering from sciatica may show a clinicoradiological discrepancy. Therefore, during the management of sciatica, the spine surgeon must overcome this discrepancy to avoid treatment failure and bad surgical results. Under such circumstances, our treatment strategy is to place even greater empha- 
Table 2. Electromyography results

\begin{tabular}{lcccc} 
& & & EMG results \\
Clinical sciatica & No. & L5 S & S1 S & NC \\
\cline { 3 - 5 } & & 2 & 3 & 5 \\
Sciatica L5 (DH L5-S1) & 10 & 4 & 1 & 3 \\
\hline
\end{tabular}

EMG, electromyography; S, sciatica; NC, non contributory; DH, disc herniation.

sis on conservative approaches and we propose epidural or periradicular infiltration to the patients. In the event of persistence of sciatica, we propose surgery, while explaining the possibility of treatment failure to the patients. Although according to our experience and based on the results of this study that confirm complete resolution of sciatica in $81.48 \%$ of patients after six weeks of surgery and in $92.59 \%$ of patients after three months of surgery, we think that patients with sciatica with disc herniation should be operated on despite this discrepancy between sciatica and disc herniation level. Obviously, the surgical decision must be taken after considering the standard of care treatment.

\section{Conflict of Interest}

No potential conflict of interest relevant to this article was reported.

\section{References}

1. Ha C, Plaine J, Fouquet N, et al. Estimation de l'incidence de la hernie discale opérée à partir des données du PMSI (France métropolitaine, 2007). Villejuif: Institut de veille sanitaire, Laboratoire d'ergonomie et d'épidémiologie en santé au travail; 2007.

2. Anema JR, Steenstra IA, Bongers PM, et al. Multidisciplinary rehabilitation for subacute low back pain: graded activity or workplace intervention or both? A randomized controlled trial. Spine (Phila Pa 1976) 2007;32:291-8.

3. Chou R, Huffman LH; American Pain Society; American College of Physicians. Medications for acute and chronic low back pain: a review of the evidence for an American Pain Society/American College of Physicians clinical practice guideline. Ann Intern Med 2007;147:505-14.
4. Chou R, Huffman LH; American Pain Society; American College of Physicians. Nonpharmacologic therapies for acute and chronic low back pain: a review of the evidence for an American Pain Society/ American College of Physicians clinical practice guideline. Ann Intern Med 2007;147:492-504.

5. Gourmelen J, Chastang JF, Ozguler A, Lanoe JL, Ravaud JF, Leclerc A. Frequency of low back pain among men and women aged 30 to 64 years in France. Results of two national surveys. Ann Readapt Med Phys 2007;50:640-4, 33-9.

6. Grellier P. Sciatique, cruralgie et canal lombaire étroit. In: Deck P, Kéravel Y, editors. Neurochirurgie. Paris: Ellipses; 1995. p.394-405.

7. Elster AD. Bertolotti's syndrome revisited. Transitional vertebrae of the lumbar spine. Spine (Phila Pa 1976) 1989;14:1373-7.

8. Mann DC, Keene JS, Drummond DS. Unusual causes of back pain in athletes. J Spinal Disord 1991;4:337-43.

9. Sabnis AB, Diwan AD. The timing of surgery in lumbar disc prolapse: a systematic review. Indian J Orthop 2014;48:127-35.

10. Truumees E. A history of lumbar disc herniation from hippocrates to the 1990s. Clin Orthop Relat Res 2014 Apr 22 [Epub]. http://dx.doi.org/10.1007/ s11999-014-3633-7.

11. Wigh RE, Anthony HF Jr. Transitional lumbosacral discs. probability of herniation. Spine (Phila Pa 1976) 1981;6:168-71.

12. Lotan R, Al-Rashdi A, Yee A, Finkelstein J. Clinical features of conjoined lumbosacral nerve roots versus lumbar intervertebral disc herniations. Eur Spine J 2010;19:1094-8.

13. Mondelli M, Aretini A, Arrigucci U, Ginanneschi F, Greco G, Sicurelli F. Clinical findings and electrodiagnostic testing in 108 consecutive cases of lumbosacral radiculopathy due to herniated disc. Neurophysiol Clin 2013;43:205-15. 